\title{
A stable scheme for computation of coupled transport and equilibrium equations in tokamaks
}

\author{
E. Fable, C. Angioni, A. A. Ivanov ${ }^{1}$, K. Lackner, O. Maj, S. \\ Yu. Medvedev ${ }^{1}$, G. Pautasso, G. V. Pereverzev $\ddagger$ \\ Max-Planck-Institut für Plasmaphysik, EURATOM Association, 85748 Garching, \\ Germany \\ ${ }^{1}$ Keldysh Institute, Russian Academy of Sciences, Moscow, Russia \\ E-mail: emiliano.fable@ipp.mpg.de
}

\begin{abstract}
The coupled system consisting of $1 \mathrm{~d}$ radial transport equations and the quasi-static $2 \mathrm{~d}$ magnetic equilibrium equation for axisymmetric systems (tokamaks) is known to be prone to numerical instabilities, either due to propagation of numerical errors in the iteration process, or due to the choice of the numerical scheme itself. In this paper, possible origin of these instabilities, specifically associated with the latter condition, is discussed and an approach is chosen which is shown to have good accuracy and stability properties. This scheme is proposed to be used within those codes for which the poloidal flux $\psi$ is the quantity solved for in the current diffusion equation. Mathematical arguments are used to study the convergence properties of the proposed scheme.
\end{abstract}

$\ddagger$ In memoriam 


\section{Introduction}

Full-discharge simulations for tokamak consist in evolving simultaneously the internal and external plasma state, which means that internal radial transport described by flux-surface averaged transport equations, and the global, free-boundary, magnetic equilibrium evolution, are coupled and evolved together [1]. Several codes exist that are capable of performing this very complex task $[2,3,4,5]$. The actuators are usually the external coil currents that are applied to shape the plasma and drive its current. Resulting plasma shape is such as to satisfy static force balance $\nabla P=\mathbf{J} \times \mathbf{B}=$ $\mathbf{J} \times(\nabla \times \mathbf{A})$, which, projected in the cross-field lines direction, gives the Grad-Shafranov equation. Simultaneously, plasma resistivity causes the poloidal magnetic field to diffuse, a process that is described by the local (purely resistive) Ohm's law $\mathbf{E}=-\frac{\partial \mathbf{A}}{\partial t}=\eta \mathbf{J}$. On the other hand $\mathbf{J}=\nabla \times \mathbf{B} / \mu_{0}$, so it is clear that it is desirable to employ a numerical scheme that allows, at each time step, to obtain consistency between the vector potential A as solved for in the Grad-Shafranov equation and in Ohm's law with the appropriate boundary conditions. Notice that the Grad-Shafranov equation is non-linear in the term $\mathbf{J} \times \mathbf{B}$, thus requiring iterative schemes for its solution. Moreover, since the complete Ohm's law contains also the 'convective' term $\mathbf{v} \times \mathbf{B}$, which is normally canceled out in transport codes via flux-surface averaging, has then to be consistent, checked 'a posteriori' with plasma motion generated in case of a free-boundary calculation. To this respect it is noted, en passant, that the electrostatic potential, being in this context equivalent to an arbitrary gauge function (as in the case of an inertial rigid motion in vacuum, where it exists purely to account for the change in reference frame), will physically adjust to bring consistency between the vectorial and the flux-surfaceaveraged Ohm's law. As such, the plasma motion consists of a sequence of quasi-static equilibria and it is not overdetermined (see Appendix A).

To abstract a bit from the physics problem itself, it is first useful to address the question of the general stability of coupled non-linear equations when iterated to obtain consistency of the solution, with respect to quantities appearing simultaneously in the dynamic (transport) and static (equilibrium) component of the coupled system. Concern for the stability is particularly severe when fast transients take place. Iteration schemes applied to reach consistency can become unstable in certain conditions.

The appearance of an instability can be generally understood by considering a coupled set of non-linear equations:

$$
\begin{aligned}
& \dot{y}=f(M, y) \\
& \mathcal{F}_{G}(M, y)=G(M, y)
\end{aligned}
$$

where the first (e.g. Ohm's law) describes time evolution of quantity $y$ (e.g. the vector potential), with quantity $M$ entering as a parameter (e.g. $M$ would be a specific metric tensor component). In the second (e.g. Grad-Shafranov), non-linear equation, $y$ acts as a 'coordinate', and $M$ in computed in terms of $y$ and by choosing an 'arbitrary' function $G(M, y)$. The specific choice of $G$ determines also the form of $\mathcal{F}$, which in 
the end must describe the same physical system. At a given time step, the fixed-point iteration defined by: $\mathcal{F}_{G}\left(M^{i+1}, y\right)=G\left(M^{i}, y\right)$ has to be solved to find the converged metric $M$. As such, a formal error estimate can be obtained by Taylor-expanding $\mathcal{F}_{G}$ in perturbations of $M$ and computing the relative change in the error $\varepsilon$ from iteration $i$ to iteration $i+1$ :

$$
\tilde{\varepsilon}=\frac{\varepsilon^{i+1}}{\varepsilon^{i}}=\frac{\left|\frac{\delta G}{\delta M}\right|_{\infty}}{\left|\left(\frac{\delta \mathcal{F}_{G}}{\delta M}\right)_{G}\right|_{\infty}}
$$

where the symbol $\delta$ indicates a derivative of functional type. A convergent scheme is characterized by $\tilde{\varepsilon}<1$, viceversa it will be unstable. It is then favourable that either the function $G$ is chosen not to depend on $M$, or that the expression for $G$ is such that the right-hand-side of equation (2) is below unity independently of the equation parameters. The method that will be presented here falls in the latter category. Notice also that achieved stability of the fixed-point iteration defined by the second of equations (1) implies stability of the full iteration scheme, including also the transport equation, if the latter adpots an implicit scheme, while for an explicit scheme it is not evident that be the case. Indeed, in the case of explicit scheme in the transport equation, the instability maybe linked to the choice of time step and the amplitude of resistive dissipation of numerically generated boundary layers.

The paper is structured as follows: in section 2 the full physical problem is discussed. In section 3 the novel numerical scheme is presented and detailed with examples. Section 4 draws the conclusions.

\section{Coupling transport and equilibrium for axisymmetric systems}

A so called 1-1/2D transport code, as routinely used in the tokamak community, solves for flux-surface-averaged conservative equations with sources and sinks [6]. As regards kinetic profiles, for example the density $n$, the equation is of the type $\frac{1}{V^{\prime}} \frac{\partial\left(V^{\prime} n\right)}{\partial t}+\frac{1}{V^{\prime}} \frac{\partial\left(V^{\prime} \Gamma\right)}{\partial \rho}=S_{n}$, with $V^{\prime}=\partial V / \partial \rho$ ( $\rho$ is a generic flux coordinate $)$, where the profile of $n(\rho, t)$ and its flux $\Gamma(\rho, t)$ evolve subjected to the boundary conditions $\Gamma(0, t)=0$ at the magnetic axis, and either $n\left(\rho_{\mathrm{b}}, t\right)=n_{\mathrm{b}}(t)$ or $\Gamma\left(\rho_{\mathrm{b}}, t\right)=\Gamma_{\mathrm{b}}(t)$ at the outer transport boundary. Resistive transport of poloidal magnetic flux is regulated by flux-surface-averaged parallel Ohm's law, $\left\langle E_{\|} B\right\rangle_{\mathrm{FS}}=\eta\left\langle J_{\|} B\right\rangle_{\mathrm{FS}}$, which turns out to be a parabolic PDE for the poloidal magnetic flux $\psi$, known as current diffusion equation (CDE). In the following the focus is put on this latter evolution equation.

The CDE is considered here in the convective-diffusive form:

$$
\left(\frac{\partial \psi}{\partial t}\right)_{x}=\frac{\eta F^{2}}{32 \pi^{2} \mu_{0} \Phi_{\mathrm{b}}^{2}} \frac{1}{x}\left[\frac{g_{3} g_{2}}{x} \psi^{\prime}\right]^{\prime}+\frac{\dot{\Phi}_{\mathrm{b}}}{2 \Phi_{\mathrm{b}}} x \psi^{\prime}
$$

where the ${ }^{\prime}=\partial / \partial x, \psi=\psi(x, t), g_{3}=\left\langle\frac{1}{R^{2}}\right\rangle_{\mathrm{FS}}, g_{2}=\left\langle\frac{|\nabla V|^{2}}{R^{2}}\right\rangle_{\mathrm{FS}}, \eta$ is the plasma resistivity, $F=R B_{\mathrm{T}}$ is the covariant component of the toroidal magnetic field, $\Phi_{\mathrm{b}}$ is the toroidal magnetic flux $\Phi$ at the boundary, $\psi$ is the poloidal magnetic flux, and 
$x=\sqrt{\Phi / \Phi_{\mathrm{b}}}$. Notice that in the temperature and density transport equations one also needs the metric coefficient $g_{1}=\left\langle|\nabla V|^{2}\right\rangle_{\mathrm{FS}}$, and some additional others for the toroidal momentum transport equation, however these metric coefficients do not enter explicitly in the iteration process described, but only parametrically in the transport equations. They can be simply updated once at the end of the equilibrium iteration process. As mentioned before, this does not cause any numerical problems in advancing the equations. As such, these additional coefficients will not be discussed any further. The operator $\langle f\rangle_{\mathrm{FS}}=\partial / \partial V \int_{0}^{V} f d V$ is the usual flux-surface-averaging. At each time step, the CDE provides a solution $\psi=\psi(x)$ subjected to appropriate boundary conditions: regularity at magnetic axis $(\partial \psi / \partial x)_{x \rightarrow 0}=0$, and at the plasma separatrix either fixed plasma current $I_{\mathrm{p}}$, i.e. $(\partial \psi / \partial x)_{x=1}=\left(4 \pi^{2} \mu_{0} I_{\mathrm{p}} /\left\langle\frac{|\nabla V|^{2}}{R^{2}}\right\rangle_{\mathrm{FS}} \partial V / \partial x\right)_{x=1}$, or imposed loop voltage $V_{\text {loop }}$, i.e. $\psi\left(\rho_{\mathrm{b}}, t\right)=\int V_{\text {loop }} d t$.

The CDE contains parametrically the metric quantities $g_{2}, g_{3}, F, \Phi_{\mathrm{b}}$, which are determined from the equilibrium equation. The spatial distribution of poloidal magnetic flux $\psi(R, Z)$, where cylindrical coordinates $(R, Z, \varphi)$ are employed is obtained via the Grad-Shafranov equation (GSE):

$$
\nabla \cdot\left(\frac{\nabla \psi}{R^{2}}\right)=-4 \pi^{2} \mu_{0} \frac{\partial P}{\partial \psi}-\frac{4 \pi^{2}}{R^{2}} F \frac{\partial F}{\partial \psi}
$$

The poloidal function $F$ is linked to $\psi$ through the relationship:

$$
F=\frac{2 \pi}{g_{3}} \frac{\partial \Phi}{\partial V}=\frac{2 \pi \Phi_{\mathrm{b}}}{g_{3}} \frac{\partial \psi}{\partial V} \hat{q}=\frac{2 \pi \Phi_{\mathrm{b}}}{g_{3}} H \hat{q}
$$

with $\hat{q}=\partial\left(x^{2}\right) / \partial \psi=q / \Phi_{\mathrm{b}}$ and $H=\partial \psi / \partial V$.

The flux-surface average of equation (4), in the plasma region, reads:

$$
\frac{\partial}{\partial V}\left(g_{2} \frac{\partial \psi}{\partial V}\right)=-4 \pi^{2} \mu_{0} \frac{\partial P}{\partial \psi}-4 \pi^{2} g_{3} F \frac{\partial F}{\partial \psi}
$$

Consistency between equation (3) and equation (4) requires that the flux $\psi$ on the $1 \mathrm{~d}$ grid $x$ and on the $2 \mathrm{~d}$ grid $(R, Z)$ mapped onto the $1 \mathrm{~d}$ grid has the same values. When this is accomplished through iterations, one can see that the most critical terms involve the quantity $g_{2}$, as from its definition it is related to local geometrical gradients. Easily that term can become unstable and lead to divergence of the iteration scheme. There have been attempts concerning possible iterations scheme and stability analysis based on equation (6) as for example in $[7,8,9,10,11,12]$. In the following section an alternative scheme is presented which is based on [8], and for which mathematical arguments are given to study its stability.

\section{A numerical scheme based on poloidal flux conservation}

Let us assume that a solution $\psi(x)$ of equation (3) has been computed, as well as a solution of the density and energy transport equations, which allow to compute a new pressure profile $P(x)$. Therefore $\psi$ is known in the whole plasma region, which allows us to immediately compute $\partial P / \partial \psi$ and $\hat{q}$. Note also that the total plasma current can 
be computed at this stage by using the new $\psi$ solution and the old values of $g_{2}$ and $F$ as $I_{\mathrm{p}}=\left(\frac{g_{2} g_{3} F}{8 \pi^{3} \mu_{0} q}\right)_{\mathrm{b}}$, which value can be used to constrain the equilibrium solution. On the other hand, the quantity $H$ is not known at this stage, since a relationship between magnetic flux and volume is provided by the GSE. To this purpose, using identity (5), equation (6) is rewritten in the form of a 1st order differential equation:

$$
H\left(g_{2} H\right)^{\prime}=-4 \pi^{2} \mu_{0} P^{\prime}-16 \pi^{4} \Phi_{\mathrm{b}}^{2} \hat{q} H\left(\hat{q}_{3} H\right)^{\prime}
$$

where $\hat{q}_{3}=\hat{q} / g_{3}$. A boundary condition is obtained by considering that, at the plasmavacuum interface $(x=1): F_{\mathrm{b}}=\left(R B_{\mathrm{T}}\right)_{\mathrm{v}}$. Employing $(5)$ to compute $H_{\mathrm{b}}$, equation $(7)$ is thus solved analytically:

$$
H=\frac{g_{2, \mathrm{~b}}+16 \pi^{4} \Phi_{\mathrm{b}}^{2} \hat{q}_{\mathrm{b}} \hat{q}_{3, \mathrm{~b}}}{g_{2}+16 \pi^{4} \Phi_{\mathrm{b}}^{2} \hat{q} \hat{q}_{3}} \sqrt{H_{\mathrm{b}}^{2} e^{[A]_{1}^{x}}+\int_{x}^{1} B P^{\prime} e^{[A]_{z}^{x}} d z}
$$

with $[A]=\int \frac{32 \pi^{4} \Phi_{\mathrm{b}}^{2} \hat{q}_{3} \hat{q}^{\prime}}{g_{2}+16 \pi^{4} \Phi_{\mathrm{b}}^{2} \hat{q} \hat{q}_{3}} d x, B=8 \pi^{2} \mu_{0} \frac{g_{2}+16 \pi^{4} \Phi_{\mathrm{b}}^{2} \hat{q} \hat{q}_{3}}{\left(g_{2, \mathrm{~b}}+16 \pi^{4} \Phi_{\mathrm{b}}^{2} \hat{q}_{\mathrm{b}} \hat{q}_{3, \mathrm{~b}}\right)^{2}}$. Note that, for the square root in equation (8) to be real, one requires a condition on the plasma pressure, which can be estimated as the condition $\beta \lesssim 1$, where $\beta$ is the ratio between plasma kinetic and magnetic pressure. For most tokamak applications $\beta$ is well below 0.1 (but for a spherical tokamak it can easily reach $\approx 0.5$, and $\beta \gtrsim 1$ means practically no confined plasma). $\Phi_{\mathrm{b}}$ appears in equation (8) as a parameter, but it has to be solved for. This is obtained by noticing that the (total) plasma volume $V_{\mathrm{b}}$ acts as a constraint:

$$
V=\int_{\psi_{0}}^{\psi} \frac{d \psi}{H\left(\Phi_{\mathrm{b}}, g_{2}\right)} \rightarrow V_{\mathrm{b}}=\int_{\psi_{0}}^{\psi_{\mathrm{b}}} \frac{d \psi}{H\left(\Phi_{\mathrm{b}}, g_{2}\right)}
$$

This constraint, together with equation (7), forms a non-linear problem which fixes both $H$ and $\Phi_{\mathrm{b}}$. At this point, the second term on the right-hand-side of equation (3) can be computed here by updating $\psi$ with the new $\Phi_{\mathrm{b}}$ and using the invariance of $q=\Phi_{\mathrm{b}} \hat{q}$. For a circular plasma with infinite aspect ratio, the quantity $g_{2} \propto V$, which is exploited to recompute the metric $g_{2}$ at each stage of the $1 \mathrm{~d}$ non-linear iteration process simply by reinterpolating $g_{2}$ as the volume changes as a function of $\psi$, thus keeping $g_{2}(V)$ (and $\left.g_{3}\right)$ fixed. The poloidal current term $F F_{\psi}$ is calculated as:

$$
F \frac{\partial F}{\partial \psi}=2 \pi^{2} \Phi_{\mathrm{b}} \frac{\partial}{\partial \psi}\left(\hat{q}_{3} H\right)^{2}=-\frac{\mu_{0}}{g_{3}} \frac{\partial P}{\partial \psi}-\frac{1}{4 \pi^{2} g_{3}} \frac{\partial}{\partial V}\left(g_{2} H\right)
$$

An alternative of the analytical solution and of the iteration scheme shown above can be derived when using the function $K=\hat{q}_{3} H$ instead of $H$, leading to $F=2 \pi K$. One advantage of using $K$ is that the boundary condition $K_{\mathrm{b}}=\left(R B_{\mathrm{T}}\right)_{\mathrm{v}} /(2 \pi)$ is independent of the boundary safety factor $q_{\mathrm{b}}$. Both formulations have been tested and the result is practically independent of which one is used, although the numerical implementation of the formulation with $K$ is actually easier and the precision to which the analytical solution is numerically represented is better. To summarize, at the end of this first iteration process identified by analytical solution (8) and the constraint (9), two things are achieved: 1) the profile of the diamagnetic function $F$ has been found, that is 
consistent with radial force balance, equation (7), together with the value of the toroidal magnetic flux at the plasma boundary $\Phi_{\mathrm{b}}$, consistent with the poloidal magnetic flux profile and the plasma volume.

The pair $(\partial P / \partial \psi, F \partial F \partial \psi)$ as functions of $\psi$ are then given as inputs to a $2 \mathrm{~d}$ fix or free-boundary Grad-Shafranov solver. The 2d GSE can be written in 1d-like form by employing flux coordinates:

$$
\frac{1}{J_{\mathrm{V}}} \frac{\partial}{\partial V}\left(J_{\mathrm{V}} \frac{|\nabla V|^{2}}{R^{2}} \mathcal{H}\right)=-4 \pi^{2} \mu_{0} \frac{\partial P}{\partial \psi}\left(1-\frac{1}{R^{2} g_{3}}\right)+\frac{1}{R^{2} g_{3}} \frac{\partial}{\partial V}\left(g_{2} H\right)
$$

where $\mathcal{H}$ is the same quantity $H$ but solved for in the $2 \mathrm{~d}$ equation (in the circular, infinite aspect ratio case one would have exactly $\mathcal{H}=H$ analytically), and $J_{\mathrm{V}}=\left(|\nabla V|\left|\nabla \theta_{\mathrm{p}}\right|\right)^{-1}$ is the Jacobian (note that the "poloidal angle" $\theta_{\mathrm{p}}$ is defined such that $\nabla V \cdot \nabla \theta \mathrm{p}=0$ ). The quantity $|\nabla V|^{2} / R^{2}$ is also solved for in the $2 \mathrm{~d}$ problem, and its solution allows to compute the new metric coefficients $g_{2}(V), g_{3}(V)$. Eventually a new plasma boundary and volume are also output of the $2 \mathrm{~d}$ problem. In the latter case it also recommended to constrain the $2 \mathrm{~d}$ solution to have the same total plasma current as given by $I_{\mathrm{p}}=\left(g_{2} H\right)_{\mathrm{b}} /\left(4 \pi^{2} \mu_{0}\right)$. To summarize, at the end of this part of the computation, that is after the solution of the $2 \mathrm{~d}$ GSE (11), the metric quantities $\left\langle\frac{|\nabla V|^{2}}{R^{2}}\right\rangle_{\mathrm{FS}},\left\langle\frac{1}{R^{2}}\right\rangle_{\mathrm{FS}}, \ldots$ are updated as the poloidal equilibrium is now satisfied.

At this stage, a convergence check can be done on the metric quantities that are output of the $2 \mathrm{~d}$ equation and the ones used to solve equation (7). If convergence is not reached, a loop is done through equation (7) and equation (11). Finally, an additional external loop will include equation (3) to achieve a full implicit solution of the coupled CDE-GSE system. This external loop does not bring in additional problems, since it features a parabolic equation of purely diffusive type, where the time step acts as an effective tuning parameter (moreover an implicit solution of equation (3) has $g_{2}$ appearing at the denominator). The pressure $P$ can also be iterated as well at the same time through its diffusion equation. The full scheme is summarized in the flow-chart of table 1.

\subsection{Stability and convergence analysis of iterative process defined by equation (9)}

Let us define $v=g_{2} / g_{2, \mathrm{~b}}=v(y), y=V / V_{\mathrm{b}}, \alpha=16 \pi^{4} \Phi_{\mathrm{b}}^{2} \hat{q} \hat{q}_{3} / g_{2, \mathrm{~b}}$, and $C=$ $\left(\sqrt{e^{[A]_{1}^{x}}+H_{\mathrm{b}}^{-2} \int_{x}^{1} B P^{\prime} e^{[A]_{z}^{x}} d z}\right) / \psi^{\prime}$. Upon using solution from equation (8), equation (9) becomes:

$$
y=G(y)=\frac{\int_{0}^{x}(v+\alpha) \frac{d x}{C}}{\int_{0}^{1}(v+\alpha) \frac{d x}{C}}
$$

The fixed-point iteration scheme $y^{i+1}=G\left(y^{i}\right)$, where $i$ is the $i$ th iteration, has the following properties: $G$ is a compact mapping from space $[0,1] \rightarrow[0,1]$, and at each iteration $i$ the solution $y$ has bounded derivatives, i.e. $\left|y^{\prime}\right|<\left|y^{\prime}\right|_{\max }$. Therefore the mapping $G$ satisfies the Arzelà-Ascoli theorem, which states that a compact mapping with absolutely bounded first derivative has at least a uniformly convergent subsequence 


\begin{tabular}{|c|c|}
\hline Step & Algorithm \\
\hline $1(\mathrm{eq}+\mathrm{tr})$ & $\begin{array}{c}\text { Advance CDE (and other transport equations) } \\
\text { from time slice }[t-d t] \text { to time slice }[t] \text { via equation }(3) \text {, } \\
\text { using } g_{2}^{i-1}, \ldots \text { as parameters } \rightarrow \psi^{i}(x, t), P(x, t) \text {. }\end{array}$ \\
\hline $2(\mathrm{eq})$ & $\begin{array}{c}\text { Uses } \psi^{i}, P, \text { and metric coefficients } g_{2}^{j-1}, g_{3}^{j-1} \\
\text { to compute } H^{j} \text { from equation }(8) .\end{array}$ \\
\hline $3(\mathrm{eq})$ & $\begin{array}{c}\text { Using } V_{\mathrm{b}} \text { as a constraint, compute new } \Phi_{\mathrm{b}} \text { from equation }(9) ; \\
\text { in addition update } g_{2}^{j-1}(\psi), g_{3}^{j-1}(\psi) \text { from new } V(\psi) \\
\text { by keeping fix dependencies } g_{2}^{j-1}(V), g_{3}^{j-1}(V) \\
\text { If needed, update } \psi \text { through adiabatic compression, } \\
\text { i.e. solve for second term in equation }(3) .\end{array}$ \\
\hline $4(\mathrm{eq})$ & Iterate $2\left(\right.$ eq) and $3($ eq $)$ until convergence in $\Phi_{\mathrm{b}}$ and $g_{2}^{j-1}(V), g_{3}^{j-1}(V)$ \\
\hline $5(\mathrm{eq})$ & $\begin{array}{c}\text { Solve for new }\left(F F_{\psi}\right)^{j} \text { as in equation }(10) . \\
\text { [Optional: apply pre-conditioning }(15) \text { on } g_{2}^{j-1} \text { to stabilize edge instability.] }\end{array}$ \\
\hline $6(\mathrm{eq})$ & Solve 2d GSE to compute new metric coefficients $g_{2}^{j}(V), g_{3}^{j}(V)$ \\
\hline $\begin{array}{l}7(\text { eq }) \\
j=j+1\end{array}$ & $\begin{array}{l}\text { [Optional: apply post-processing (16) on } g_{2}^{j} \text { to speed-up convergence.] } \\
\text { Restart from } 2(\mathrm{eq}) \text { until convergence in metric coefficients. }\end{array}$ \\
\hline $\begin{array}{c}8(\mathrm{eq}+\mathrm{tr}) \\
\mathrm{i}=\mathrm{i}+1\end{array}$ & $\begin{array}{l}\text { Update } g_{2}^{i}=g_{2}^{j}, \ldots \\
\text { Iterate with } 1(\mathrm{eq}+\mathrm{tr}) \text { until convergence on } \psi(x, t) \text { is reached. }\end{array}$ \\
\hline
\end{tabular}

Table 1. Flow-chart of the proposed algorithm. Index $i$ refers to external iterations with CDE (transport equations 'tr'), while index $j$ refers to iterations in the equilibrium part 'eq'. Note that $g_{2}^{i=1}=g_{2}(t-d t)$, and so on for other geometrical quantities. Also $g_{2}^{j=1}=g_{2}^{i-1}, \ldots$. The steps in square parenthesis and marked with 'Optional' are not necessary for the method to work, but are suggested to remedy to the appearance of the instability discussed in section 3.2 .

$\S$. The compactness of mapping $G$ is trivially proven following its definition. The proof of the absolute boundedness of the first derivative can be obtained by considering that $y^{\prime}=(v+\alpha) /(A C)$, where $A=\int_{0}^{1}(v+\alpha) \frac{d x}{C}$. First, $\min (C)$ is obtained when $v=1$. For $A$, i.e. $\min (A)$ can be considered independent of $v$, since the maximal $C$ is obtained when $v=0$. In conclusion, one finds that $\max \left(\left|y^{\prime}\right|\right)=[1+\max (\alpha)] /\left[A_{v=0} C_{v=1}\right]$ and that it is independent of $y$, and then the absolute maximum bound on the first derivative of the mapping $G$ is the same for all functions $y$ in the iteration procedure. It is possible, but very lengthy, to prove that the sequence $G^{i}$ is a Cauchy sequence, i.e. discrepancy between following iterations goes to zero for sufficiently large $i$. Therefore, if the sequence $G^{i}$ contains convergent subsequences, and converges to some value for large $i$, this means that the value is unique and the sequence converges to that solution

$\S$ http://en.wikipedia.org/wiki/Arzel\%C3\%A0\%E2\%80\%93Ascoli_theorem . Note that this theorem does not guarantee that the series itself will numerically converge or that the converged value is unique. 
$\|$. The detailed proof of this statement is given in Appendix B.

It is necessary mentioning here that, in the case where relevant quantities, as $\hat{q}, g_{2}, \ldots$, logarithmically diverge at the separatrix, e.g. in case of a X-point configuration, then the procedure describes above makes sense when the location of the equilibrium 'boundary' $x=1$ is considered as neighboring the separatrix but not coinciding.

\subsection{Stability analysis of iterative process including equation (11)}

Equation (11) is formally solved for with prescribed right-hand-side on flux coordinates to find both the Jacobian $J_{\mathrm{V}}$ and the unknown functions $\mathcal{H},|\nabla V|^{2} / R^{2}$.

By taking the flux-surface average of equation (11), and calling $G_{2}=\left\langle|\nabla V|^{2} / R^{2}\right\rangle$ the same metric coefficient as $g_{2}$ but computed in the $2 \mathrm{~d}$ equation, the relation is found:

$$
G_{2} \mathcal{H} \approx g_{2} H
$$

where the approximate symbol is due to $g_{3}$ being not exactly equal to the flux surface average of $1 / R^{2}$ from the $2 \mathrm{~d}$ equation (they become equal at convergence). Relation (13) allows an easier formal estimate of the iteration error from equation (2):

$$
\tilde{\varepsilon}=\frac{\varepsilon^{i+1}}{\varepsilon^{i}} \approx \frac{\left|H+g_{2}\left(\frac{\delta H}{\delta g_{2}}\right)\right|_{\infty}}{\left|\mathcal{H}+G_{2}\left[\frac{\delta \mathcal{H}(\delta j)}{\delta G_{2}(\delta j)}\right]\right|_{\infty}}
$$

where $\delta \mathcal{H} / \delta G_{2}$ has to be interpreted as the ratio of the two variations of the functionals $\mathcal{H}, G_{2}$ as functions of the source $j$. The quantity $j$ indicates the known source, or right-hand-side of the GSE (i.e. the toroidal current density). Notice that $\frac{\delta H}{\delta g_{2}}<0$, as such one sees that the denominator is the critical factor in determining the convergence properties of the scheme. In general the dependence of the flux-surface geometry on the source is expected to be weak, an example being the well known Solov'ev solution [13], for which the impact of the source is only in determining the magnetic axis position (Shafranov-shift). However it is difficult a priori to assess the actual impact of the source when a numerical solution is computed. From equation (14) it is clear that an instability can only appear provided an inverse dependence of $G_{2}$ on $j$, which results in $\delta G_{2} / \delta j<0$, while in general one finds that $\delta H / \delta j>0$.

Let us first tackle a situation in which the plasma boundary has no singular points, i.e. it is defined by a regular curve (no X-points present). In this case the geometry of the flux surfaces is not so sensitive on the source, even in the numerical solution, i.e. $G_{2}$ does not depend on $j$, which leads to $\tilde{\varepsilon} \rightarrow 0$. Therefore convergence will be reached rapidly as $g_{2} \rightarrow G_{2}$ after a few iterations.

Problems arise when dealing with a plasma with field singular points on the boundary (the suspect is that actually boundary regions with very large local curvature are the most fragile). In the case of an $\mathrm{X}$-point, in its vicinity the quantity $\mathcal{H}$

\| This is a corollary to the Arzelá-Ascoli theorem if the sequence under consideration is also a Cauchy sequence. 
goes to zero as $\mathcal{H} \sim-1 / \log (\Delta)$, where $\Delta$ is the distance from the $\mathrm{X}$-point in a direction perpendicular to field lines in the plasma [14]. One can actually estimate $\mathcal{H} \sim-j / \log (\Delta)$, with $j$ the local toroidal current density. This means that relation (13) is satisfied if $G_{2} \sim-\log (\Delta) g_{2} H / j$. In a numerical solution, $\Delta$ will always have be a finite number, as such the updated $G_{2}$ will be strongly dependent on $j$, thus the $G_{2} \sim 1 / j$ dependence makes $\tilde{\varepsilon} \rightarrow \infty$, rapidly leading to a numerical divergence. Since close to the boundary the product $G_{2} \mathcal{H} \propto I_{\mathrm{p}}$, the instability appears as an oscillation of both $G_{2}$ and $\mathcal{H}$ in counterphase, which points describe a hyperbole in the $\left(G_{2}, \mathcal{H}\right)$ plane, and which increases in excursion as iterations progress.

The most straightforward way to suppress the instability is to impose a monotonicity constraint on $G_{2}$, although it is not evident that this be the general situation. It is also found that extrapolation to the separatrix of the metric coefficients values can have an impact on the strenght of this oscillation. A linear extrapolation is used, which strongly mitigates the $G_{2}$ excursions at the boundary as compared for example to quadratic extrapolation. Under-relaxation is then employed (a weighting factor of 0.5 is found to be sufficient in all tested cases, but maybe not sufficient in extreme cases).

An alternative method is proposed here which exploits equation (14) in this way: the function $g_{2}$ is multiplied by a test function $\hat{f}$ that is strongly localized near the plasma edge, and is such that $g_{2} \sim 1 / H$, i.e. making the numerator of equation (14) vanish. The test function becomes weaker as $\varepsilon^{i}$ decreases, relaxing $g_{2}$ to the real solution. The relaxation process is sped-up by employing under-relaxation factor of 0.5 . In practice, a $\tilde{g}_{2}$ is computed as:

$$
\tilde{g}_{2}=g_{2}+\hat{f} \frac{\varepsilon e^{D \varepsilon}}{1+\varepsilon e^{D \varepsilon}}\left(\frac{g_{2, \mathrm{~b}} H_{\mathrm{b}}}{H}-g_{2}\right)
$$

where $1 \ll D<1 / \varepsilon_{\text {tol }}$ ( $\varepsilon_{\text {tol }}$ is the requested tolerance). $\tilde{g}_{2}$ is then used to compute the right-hand-side in equation (11). The choice of the test function $\hat{f}$ is at this stage arbitrary and could be chosen differently. An example that has been tested is $\hat{f}=x^{4} e^{-3\left(1-x^{4}\right)}$ which seems to work fine. The exponential factor is such that for, $\varepsilon \gg 1 / D$, then $g_{2} \sim 1 / H$ at the plasma edge, while for $\varepsilon \sim D$, the correction to $g_{2}$ scales as $\varepsilon$. This method forces the numerical solution to maintain a relative error between iterations which is less or of the order of $1 / D$, thus effectively removing the instability. Note also that $\tilde{g}_{2, \mathrm{~b}}=g_{2, \mathrm{~b}}$, thus leaving the total plasma current $I_{\mathrm{p}} \propto\left(g_{2} H\right)_{\mathrm{b}}$ unchanged.

By taking the spatial derivative of relation (13), i.e. $G_{2}^{\prime} \mathcal{H}+G_{2} \mathcal{H}^{\prime} \approx g_{2}^{\prime} H+g_{2} H^{\prime}$, and replacing quantities in this form: $\tilde{G}_{2}^{\prime} \mathcal{H}_{i}+G_{2, i} \frac{\mathcal{H}_{i-1}^{\prime}}{\mathcal{H}_{i-1}} \mathcal{H}_{i} \approx G_{2, i}^{\prime} \mathcal{H}_{i}+G_{2, i} \mathcal{H}_{i}^{\prime}$, one can compute

$$
\tilde{G}_{2}=G_{2, i}+\lambda \int_{0}^{x} G_{2, i}\left[\frac{\mathcal{H}_{i}^{\prime}}{\mathcal{H}_{i}}-\frac{\mathcal{H}_{i-1}^{\prime}}{\mathcal{H}_{i-1}}\right] d x
$$

where $i$ indicates the present iteration number while $i-1$ is the previous iteration. $\lambda$ is a numerical constant that regulates the weight of the correction, with $\lambda \leq 1$, in the 
following examples the values $\lambda=0.1$ (at present this value is deduced from various testings and not from first principles arguments). $\tilde{G}_{2}$ will be used as the new metric at the next iteration, such that any $G_{2} \propto 1 / \mathcal{H} \rightarrow G_{2}^{\prime} \propto-G_{2} \mathcal{H}^{\prime} / \mathcal{H}$ dependency is somewhat removed, making the denominator of equation (14) of order 1.

Patching together the $g_{2}$ 'pre-conditioning' through equation (15) and the $G_{2}$ 'post-processing' through equation (16), it is found that the instability associated with boundary effects is strongly suppressed (by 'pre-conditioning'), and that convergence speed is improved (by 'post-processing'). Note however that the tolerance cannot be lower than the natural numerical discrepancy between $H$ and $\mathcal{H}$ as compute by two different codes (the 1d equation solved 'analytically' while the 2d GSE is solved by a specific GS code). In the following examples, the simple remedy of setting underrelaxation and linear extrapolation to the boundary works for all cases. The proposed, mathematically-based patches discussed previously might be invoked when treating more extreme configurations where simple remedy fails, as it will be shown in a specific case in the following examples section. Nonetheless, in the next section it is also shown how the number of iterations is influenced by these patches in conditions encountered typically.

\subsection{Examples}

The equilibrium code SPIDER [15] is used to perform the various testings. It has been embedded in the iteration scheme previously described and via this method coupled to the ASTRA transport code [16]. SPIDER solves the prescribed boundary problem on an adaptive, flux-coordinate grid.

The test cases are of five types: (1) static with high edge safety factor (low plasma current), (2) rapidly evolving plasma shape (elongation increase) in elliptic configuration, (3) high-beta, high edge bootstrap current case, (4) full transport (all channels evolving with prescribed parabolically-shaped diffusivities) in limiter-diverted configuration change, (5) a free-boundary computation of a spontaneous Vertical Displacement Event (VDE) in ASDEX Upgrade, with fixed kinetic profiles but evolving poloidal magnetic flux. A tolerance of $10^{-5}$ on convergence check is set for the various quantities. The iteration scheme includes iterations on the equilibrium side as well as the external loop with the CDE. Cases 1-4 are based on a reference which is a typical current ramp-up scenario in the ASDEX Upgrade tokamak [17]. The different parameters used in the simulations are listed in table 2. As regards the numerical discretization, the $2 \mathrm{~d}$ grid in SPIDER has 51 radial and 43 poloidal points, while ASTRA has 201 radial grid points for the solution of the CDE. In all cases the time step is $d t=1 \mathrm{~ms}$.

For case 1, a glance at the number of iterations required to reach consistency is shown in figure $1(\mathrm{a}, \mathrm{b}, \mathrm{c})$. The relative error on $g_{2}$ is shown in solid lines as a function of the iteration number (cumulated over time steps). Three methods are compared: Method A (black line) is skipping the scheme presented in this paper and computes $F F^{\prime}$ in particular from the formula $F F^{\prime}=-\frac{F \mu_{0}}{\left\langle B^{2}\right\rangle_{\mathrm{FS}}}\left(\frac{\langle\mathbf{J} \cdot \mathbf{B}\rangle_{\mathrm{FS}}}{2 \pi}+F \frac{\partial P}{\partial \psi}\right)$, at each iteration 


\begin{tabular}{|c|c|}
\hline Case & Relevant parameters \\
\hline 1 & $I_{\mathrm{p}}=0.44 \mathrm{MA}, q_{95} \approx 13$, peaked $T_{\mathrm{e}}, n_{\mathrm{e}}$ profiles, X-point present \\
\hline 2 & $k_{t=0}=1, k_{t=\infty} \rightarrow 1.7, d k / d t=3.5[1 / \mathrm{s}], I_{\mathrm{p}}=0.44 \mathrm{MA}$, no X-point \\
\hline 3 & $f_{\mathrm{bs}} \approx 0.5, \beta_{\text {tor }} \approx 3.3 \%, q_{95} \approx 5.6, I_{\mathrm{p}}=1 \mathrm{MA}, \mathrm{X}-$ point present \\
\hline 4 & evolving $T_{\mathrm{e}}, T_{\mathrm{i}}, n_{\mathrm{e}}, \mathrm{iL} \rightarrow$ LSN configuration change with $I_{\mathrm{p}}=0.4 \mathrm{MA}$ \\
\hline 5 & evolving $\psi$, downward VDE at $I_{\mathrm{p}}=0.8 \mathrm{MA}$ in LSN configuration \\
\hline
\end{tabular}

Table 2. Characterization of the different cases in terms of parameters change and peculiarities. "iL" = inner limited, "LSN" = lower single null configuration. X-point is always in LSN. For all cases $\psi$ and equilibrium are evolved at each time step $(d t=1$ $\mathrm{ms})$.

with the CDE. Method B (blue line) is the scheme presented here, where CDE is iterated with equilibrium, however only one equilibrium call is performed at each iteration on the CDE. Method C (red line) is also iterating the equilbrium calls with the pre and post-processing of formulas (15) and (16). The time steps advance are also shown in dashed lines with same colour coding. Three plots (a), (b), (c) are obtained by using as time step $d t=1 \mathrm{~ms}(\mathrm{a}), d t=0.1 \mathrm{~ms}(\mathrm{~b})$, and $d t=0.01 \mathrm{~ms}$ (c). From the comparison of the different plots, it is clear that the method presented here (either Method B or $\mathrm{C}$ ) is robust and reliable with respect to Method A which, instead of solving the FSAGSE, uses the parallel current as input (this was actually the method previously used in ASTRA). As it can be seen by reducing time step, Method A also can result in very slow or non-convergence at all. Note also (not shown here) that pre-conditioning (15) and post-processing (16) increase convergence speed for Method C. Using the full Method $\mathrm{C}$, time evolution requires very few external iterations per time step, in this case about $3-4$, while each external iteration contains $\approx 6-8$ equilibrium iterations on the metric coefficient, for a total of $\approx 20-30$ equilibrium calls per time step. In principle Method $\mathrm{B}$, which calls equilibrium only once per CDE call, is the fastest, although it has to be mentioned here that its stability relies also on the fact that the CDE is advanced using an implicit time scheme. If the CDE was advanced with an explicit time scheme, it is very well possible that Method B would not converge in some situations.

For the case 2 with strong elongation increase, figure 2 shows time traces of the elongation $k$, the computed $q_{95}$, and the computed boundary poloidal flux $\psi_{\mathrm{b}}$. About 20 iterations per time step are required to reach full implicit convergence of geometry and magnetic fluxes, of which 2-3 external iterations with the CDE and 7-8 iterations for geometry convergence, i.e. a total of $\approx 14-24$ equilibrium calls per time step. Notice that, after a time step in CDE is performed, the relative error at the beginning of the iteration process is $\approx 10^{-3}$, that is two orders of magnitude larger than the target tolerance. This behaviour is the same as observed in figure 1(a), since the time stepping used is the same ( $d t=1 \mathrm{~ms}$ ) and the current diffusion process is changing the poloidal flux $\psi(\rho)$ in a similar fashion.

The high- $\beta$ plasma, case 3 , is characterized by a strong Shafranov-shift of $\approx+10$ 
$\mathrm{cm}$, as shown in figure 3(a), resulting from a large edge boostrap current contribution to the total plasma current density, as shown in figure 3(b). In the same figure 3(b) also the quantity $F=R B_{\mathrm{T}}$ is plotted, which shows a strong dip in the whole plasma region, which thus behaves diamagnetically, as opposed to low- $\beta$ scenarios which which behave usually paramagnetically. Note that the edge current has still a rather substantial (and negative) Ohmic contribution, as such the total parallel current is somewhat lower than the bootstrap current.

The case 4 is characterized by evolution of all plasma profiles and transition from limited to $\mathrm{X}$-point configuration. The two flux surfaces shape corresponding to initial and final state are compared in figure $4(\mathrm{a})$. The transition takes place around $t \approx 0.1$ s. Time traces of boundary loop voltage $V_{\text {loop }}$, elongation $k$, and plasma current $I_{\mathrm{p}}$ are shown in figure 4(b). The loop voltage displays many features that are caused by the evolving $T_{\mathrm{e}}, k$, and $I_{\mathrm{p}}$. As in case 2 , about a total 20 iterations per time step are needed to reach full convergence (6-7 iterations in the equilibrium part, and 2-3 external iterations with the CDE).

Final case 5 features a spontaneous, downward VDE obtained by freezing the active feedback control coils at $t \approx 3.02 \mathrm{~s}$. The passive stabilizer coils time scale is $\tau_{\mathrm{PSL}} \sim 0.5 \mathrm{~s}$, while the vacuum vessel time scale is much shorter $\approx 20 \mathrm{~ms}$. The simulation is carried out until the plasma displacement $\delta Z \approx-15 \mathrm{~cm}$, after which the code starts to take longer to converge. Each time step takes a total of about 20 iterations to converge. Again a 5-7 iterations are taken in the equilibrium side, while 2-3 iterations are required to converge with the CDE too. Note also that, for the circuit equations, which are solved iteratively in SPIDER, a tolerance of $10^{-5}$ is set as well on the convergence between changes in coil currents and the plasma motion induced by them. In figure 5(a) the time traces of vertical position of the magnetic axis $Z$ are compared between the CLISTE equilibrium reconstruction code [18] and the simulation from ASTRA-SPIDER. As it can be seen, a good agreement is found. The istantaneous time scales $\tau=|\delta Z d t / d(\delta Z)|$ are compared in figure $5(\mathrm{~b})$, where it is shown that the rise phase and the late acceleration phase are qualitatively well reproduced. Notice that the total number of iterations $\approx 20$ mentioned above is actually observed to steadily increase when the plasma enters the non-liner acceleration phase (large vertical plasma displacement) or alternatively the time step could be reduced to maintain the same number of iteration per time step.

\section{Conclusions}

Upon employing a specific form of the flux-surface averaged GSE and choosing to consider $\psi(x)$ as a conserved quantity during the iteration process, a scheme has been proposed which does a good job in several plasma configurations.

The convergence of the $1 \mathrm{~d}-2 \mathrm{~d}$ coupled system is such that self-consistency of metric quantities and diffusing poloidal flux is assued, so that an additional external loop with the CDE itself does not produce any instability. 
The coupled flux-surface averaged and 2d GSE equations are effectively the same physical entity describing radial and poloidal force balance. Convergence of the metric at this stage means that there is no residual discrepancy of geometrical quantities once the CDE is called for the successive iteration or time step.

Mathematical arguments are put forward to prove intrinsic stability of the $1 \mathrm{~d}$ iteration scheme that computes the new toroidal magnetic flux and updates metric coefficients as functions of the plasma volume, decoupling them from the magnetic flux coordinate and thus obtaining a speed-up of the whole iteration process. Analysis of the error estimate allows to construct a relaxation scheme such that successive iterations with the 2d GSE solver are stabilized in presence of non-circular geometry with singular points.

The shown examples demonstrate this efficiently for a variety of plasma configurations and grid resolution. In particular, cases with very high edge safety factor, $\mathrm{X}$-point configuration, large edge bootstrap current, rapidly varying plasma conditions, and in the free-boundary computation of a VDE, all have passed the test and give confidence in the method.

Benchmarking the ASTRA-SPIDER code coupling against third-party codes is also planned, but yet not performed, as it will require dedicated time to select a standard case and carry out the set of simulations.

Future applications of the proposed scheme will focus on full-discharge freeboundary computations with self-consistent evolution of the external currents under feedback control, of the magnetic fluxes and of the plasma kinetic profiles.

Acknowledgments One of the authors (E.F.) is thankful to the anonymous Referees for the comments that have helped improving the manuscript. Useful discussions with Dr. M. Ottaviani are also acknowledged.

\section{References}

[1] J. Blum and J. Le Foll, Computer Physics Reports 1, 465 (1984)

[2] S. C. Jardin et al., J. Comput. Phys. 66, 481 (1986)

[3] J. A. Crotinger et al., "Corsica; A comprehensive simulation of toroidal magnetic-fusion devices", Technical Report UCRL-ID-126284, Lawrence Livermore National Laboratory (1997)

[4] R. R. Khayrutdinov et al., Plasma Phys. Control. Fusion 43, 321 (2001)

[5] M. Honda, Comput. Phys. Commun. 181, 1490 (2010)

[6] F. L. Hinton and R. D. Hazeltine, Rev. Mod. Phys. 48, No. 2, Part I (1976)

[7] H. Grad and J. Hogan, Phys. Rev. Lett. 24, 1337 (1970)

[8] H. Grad et al., Proc. Natl. Acad. Sci. USA 72, 3789 (1975)

[9] J. A. Holmes et al., J. Comput. Phys. 36, 35 (1980)

[10] J. Blum et al., Comput. Phys. Commun. 24, 235 (1981)

[11] L. L. LoDestro and L. D. Perlstein, Phys. Plasmas 1, 90 (1994)

[12] D. E. Shumaker, J. Comput. Phys. 53, 456 (1987)

[13] L. S. Solov'ev, Zh. Eksp. Teor. Fiz. 53, 626 (1976)

[14] Shi Bing-Ren, Chin. Phys. B 21, 045203 (2012)

[15] A. A. Ivanov et al., 32nd EPS Conf. on Plasma Phys., ECA Vol.29C, P-5.063 (2005) 
[16] G. V. Pereverzev et al., IPP Report 5/42 (August 1991)

[17] E. Fable et al., Nucl. Fusion 51, 043006 (2011)

[18] P.J. Mc Carthy et al., Plasma Phys. Control. Fusion 54, 015010 (2012)

[19] E. A. Lazarus and G. H. Neilson, Nucl. Fusion 27, 383 (1987)

[20] E. A. Lazarus et al., Nucl. Fusion 30, 111 (1990)

[21] A. H. Boozer, Phys. Fluids 24, 1999 (1981) 


\section{Appendix A}

It is shown here that, if the plasma motion is described by a series of equilibria consistent with external currents distribution, then consistency of Ohm's law requires a scalar potential of Pfirsch-Schlüter type, and thus velocity field is not overdetermined.

Let us define the simplified problem of a force-free plasma, where plasma motion is determined by the equation $M n \dot{\mathbf{v}}=\mathbf{J} \times \mathbf{B}$, where $M, n$ are respectively the plasma mass and particle density, and $\dot{\mathbf{v}}=d \mathbf{v} / d t=\partial \mathbf{v} / \partial t+\mathbf{v} \cdot \nabla \mathbf{v}$ is the Lagrangian acceleration. The current can thus be formally solved for to be $\mathbf{J}=j_{\|} \mathbf{B} / B-M n \dot{\mathbf{v}} \times \mathbf{B} / B^{2}$. From quasi-neutrality $\nabla \cdot \mathbf{J}=0$ one also sees that $\frac{j_{\|}}{B}+\frac{M n B_{\mathrm{T}}}{B^{2}}(\dot{\mathbf{v}})_{r}$ is a flux funtion. At each time slice, the plasma motion has to be consistent with the $\mathbf{B}$ field as a sum of the one created by the external currents distribution and by the plasma current. It is then obvious that, given two successive time slices, the displacement vector associated with the motion of the field lines is at this stage independent of Ohm's law. However the latter has to be consistent with the field lines displacement, as it is demonstrated below.

Ohm's law is $\mathbf{E}+\mathbf{v} \times \mathbf{B}=\eta \mathbf{J}$. The field $\mathbf{v}$ is related to the displacement of the plasma particles (electrons and ions). The electric field is represented in terms of the four-potential $(\mathbf{A}, \phi)$ as $\mathbf{E}=-\frac{\partial \mathbf{A}}{\partial t}-\nabla \phi$. The potential vector $\mathbf{A}$ is orthogonally decomposed via the magnetic fluxes: $\mathbf{A}=\Phi \frac{\nabla \theta}{2 \pi}-\psi \frac{\nabla \varphi}{2 \pi}+\nabla \chi$, where $\theta(\mathbf{x}, t)$ is the so called 'poloidal' angle, that satisfies $\nabla \theta \cdot(\nabla \psi, \nabla \varphi)=0$, and $\chi$ is a yet-to-be-specified gauge function. Note also that, while $\theta$ and $\phi$ are both multi-valued functions of $\mathbf{x}$, due to the arbitrariness in the offset in terms of multiple $2 \pi$ turns, their differentials $(\nabla, \partial / \partial t)$ are instead well behaved. Upon substitution of the vector potential expression into Ohm's law and by taking the toroidal and poloidal projections of the equation, one arrives at the following relations $\left(\mathbf{v}_{\theta} \cdot \nabla \theta \equiv-\frac{\partial \theta}{\partial t}\right)$ :

$$
\begin{aligned}
& \frac{\partial \psi}{\partial t}+\mathbf{v}^{*} \cdot \nabla \psi=2 \pi \eta F \frac{j_{\|}}{B} \\
& \frac{\mathbf{B} \cdot \nabla \theta}{2 \pi}\left[\frac{\partial \Phi}{\partial t}+\mathbf{v}^{*} \cdot \nabla \Phi\right]+\mathbf{B} \cdot \nabla\left[\phi+\frac{\partial \chi}{\partial t}-\frac{\Phi}{2 \pi} \mathbf{v}_{\theta} \cdot \nabla \theta\right]=-\eta j_{\|} \frac{B_{\mathrm{p}}^{2}}{B}
\end{aligned}
$$

where $\mathbf{v}^{*}=\mathbf{v}+\frac{M n \eta}{B^{2}} \dot{\mathbf{v}}$. It has been shown in previous studies, see e.g. [19, 20], that, if certain conditions related to the balance between magnetic forces which the plasma feels are satisfied, then the Alfvén branch of the plasma motion is stable, i.e. it is rapidly damped. On the other hand, the resistive branch, associated with currents induced in the conducting structures, is unstable. This means that one can estimate $\dot{\mathbf{v}} \sim \mathbf{v} / \tau_{\mathrm{RL}}$, where $\tau_{\mathrm{RL}} \sim L / R$ is a typical resistive time scale associated with the conducting structures. As such, one is allowed to neglect the term $\frac{M n \eta}{B^{2}} \dot{\mathbf{v}}$, as compared to $\mathbf{v}$, if the Chandrasekhar number $C_{\mathrm{h}}=\frac{B^{2} \tau_{\mathrm{RL}}}{M n \eta} \gg 1$. As an example, taking typical plasma conditions $n \sim 10^{19}$ $\mathrm{m}^{-3}, M \sim 3 \cdot 10^{-27} \mathrm{~kg}, B \sim 2 \mathrm{~T}, \eta \sim 10^{-6} \Omega \mathrm{m}$, and $\tau_{R L} \sim 20 \mathrm{~ms}$, one gets $C_{\mathrm{h}} \sim 10^{12}$. 
Moreover, one can also see that $\frac{j_{\|}}{B}$ becomes a flux function. In the following it is then possible to set $\mathbf{v}^{*} \rightarrow \mathbf{v}$ and $\frac{j_{\|}}{B}$ as a flux function.

Since the FSA of Ohm's law is nothing else than the CDE, equation (3), and manipulating via chain derivative rule, one obtains the solubility condition of equations (17):

$$
\frac{\mathbf{B} \cdot \nabla \theta}{2 \pi}\left(\frac{\partial \Phi}{\partial t}\right)_{\psi}+\mathbf{B} \cdot \nabla\left[\phi+\frac{\partial \chi}{\partial t}-\frac{\Phi}{2 \pi} \mathbf{v}_{\theta} \cdot \nabla \theta\right]=-\eta j_{\|} B
$$

This equation is independent of the velocity field $\mathbf{v}$, and requires $\phi$ as its solution $\left(V^{\prime}=\partial V / \partial \psi\right)$ :

$$
\phi(\psi, \theta)=\phi_{0}(\psi)-\frac{\eta j_{\|}}{B} \int_{0}^{\theta}\left[B^{2}-\frac{\mathbf{B} \cdot \nabla \theta}{2 \pi} V^{\prime}\left\langle B^{2}\right\rangle_{\mathrm{FS}}\right] \frac{d \theta}{\mathbf{B} \cdot \nabla \theta}
$$

where now the gauge $\frac{\partial \chi}{\partial t}=\frac{\Phi}{2 \pi} \mathbf{v}_{\theta} \cdot \nabla \theta$ is fixed. Note that other gauges are possible, e.g. $\chi=0$, which leads to $\phi=\phi_{0}-\frac{\Phi}{2 \pi}\left(\mathbf{v}_{\theta} \cdot \nabla \theta-\left[\mathbf{v}_{\theta} \cdot \nabla \theta\right]_{\theta=0}\right)-$ $\frac{\eta j_{\|}}{B} \int_{0}^{\theta}\left[B^{2}-\frac{\mathbf{B} \cdot \nabla \theta}{2 \pi} V^{\prime}\left\langle B^{2}\right\rangle_{\mathrm{FS}}\right] \frac{d \theta}{\mathbf{B} \cdot \nabla \theta}$. Substituting back the solution obtained for the scalar potential in the second of equation (17), one obtains:

$$
\frac{\partial \Phi}{\partial t}+\mathbf{v} \cdot \nabla \Phi=\left(\frac{\partial \Phi}{\partial t}\right)_{\psi}+2 \pi \eta \frac{j_{\|}}{B} F \frac{\mathbf{B} \cdot \nabla \varphi}{\mathbf{B} \cdot \nabla \theta}
$$

and finally $\left(\mathbf{v}_{\phi} \cdot \nabla \Phi \equiv-\frac{\partial \Phi}{\partial t}, \Psi=\psi / 2 \pi\right)$ :

$$
\left\langle\left(\mathbf{v}-\mathbf{v}_{\phi}\right) \cdot \nabla \Psi\right\rangle_{\mathrm{FS}}=\eta \frac{j_{\|}}{B} F\left(1-\frac{\left\langle B^{2}\right\rangle_{\mathrm{FS}}}{F^{2}} \frac{1}{2 \pi} \oint R^{2} d \theta\right)
$$

It is then straighrfoward to compare this with the usual expression of (one of the contributions to) the Pfirsch-Schlüter particle flux, but note that in usual situations at finite pressure gradient $P^{\prime}$ the dominant Pfirsch-Schlüter contribution is the one proportional to $P^{\prime}$ (see e.g. $[6,21]$ ).

\section{Appendix B}

In the following it is demonstrated that equation (12) defines a stable iteration scheme. The demonstration uses a few steps.

A simpler fixed-point theorem

Here it is demonstrated that the model fixed-point iteration given by the following relation:

$$
y^{i+1}(x)=\frac{\int_{0}^{x}\left(y^{i}(x)+\alpha\right) d x}{\int_{0}^{1}\left(y^{i}(x)+\alpha\right) d x}
$$

when subjected to the conditions $0 \leq y \leq 1, \alpha>0$ a constant, is a uniformly convergent series with a unique solution. As such, whichever the initial guess $y^{0}(x)$, the series will always converge to the unique solution $y(x)$. 
First of all, notice that the analytical solution can be found simply upon differentiation of equation (22): $y^{\prime}=A(y+\alpha)$, where $A=1 / \int_{0}^{1}(y+\alpha) d x$. The solution is thus $y=\alpha\left(e^{A x}-1\right)$, where consistency requires $A=\log (1+1 / \alpha)$, which is satisfied by the solution. Thus the analytical solution is $y=\alpha\left[\left(\frac{1+\alpha}{\alpha}\right)^{x}-1\right]$. It has been just shown that fixed-point iteration (22) has a unique, analytical solution. To demonstrate that it also converges to it uniformly, number theory is employed.

Lengthy algebra which involves successive substitutions of the formal solution into new iteration, leads to the following recursive formula, which gives the solution $y$ at iteration $N, y_{N}=B_{N} / A_{N}$, where:

$$
\begin{aligned}
& B_{N}=\tilde{g}_{N}+\sum_{q=1}^{N} \alpha^{q} \sum_{M=q-1}^{N-i_{1}} \sum_{i_{1}=1}^{N-q+1} \frac{x^{i_{1}}}{i_{1} !} \frac{\sigma(M, q-1)}{M !} \overline{\tilde{g}}_{N-M-i_{1}-1} \\
& A_{N}=\overline{\tilde{g}_{N-1}}+\sum_{q=1}^{N} \alpha^{q} \sum_{M=q-1}^{N-i_{1}} \sum_{i_{1}=1}^{N-q+1} \frac{1}{i_{1} !} \frac{\sigma(M, q-1)}{M !} \bar{g}_{N-M-i_{1}-1}
\end{aligned}
$$

$g$ is the initial guess. The notation is the following: $\tilde{g}_{N}$ indicates $N-1$ integrations in $x=0 . .1$ and one last integral from 0 to $\mathrm{x}, \overline{\tilde{g}}_{N}$ means $N$ integrations from 0 to 1 .

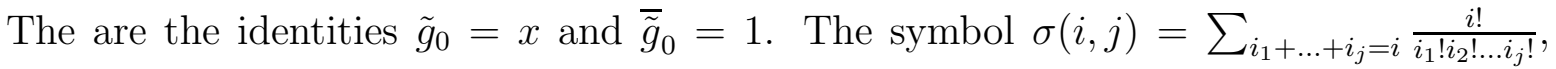
with all integer numbser strictly positive, is known in combinatorics as the number of surjections, and it is related to Stirling numbers $\Phi$. It has the property that $\sigma(a, 0)=1$ and $\sigma(a, b)=0$ if $a<b$. The Stirling number of the first kind $S(a, b)$ is defined as $S(a, b)=\sigma(a, b) / b$ !

One notes immediately that: $\sum_{M=q-1}^{N-i_{1}} \sum_{i_{1}=1}^{N-q+1} \frac{1}{i_{1} !} \frac{\sigma(M, q-1)}{M !} \overline{\tilde{g}_{N-M-i_{1}-1}}=$

$\sum_{P=i_{1}+q-1}^{N} \sum_{i_{1}=1}^{N-q+1} \frac{1}{i_{1} !} \frac{\sigma\left(P-i_{1}, q-1\right)}{\left(P-i_{1}\right) !} \bar{g}_{N-P-1}=$

$\sum_{P=M}^{N} \sum_{M=q}^{P} \frac{1}{(M-q+1) !} \frac{\sigma(P-M+q-1, q-1)}{(P-M+q-1) !} \overline{\tilde{g}_{N-P-1}}=$

$\sum_{P=q}^{N} \sum_{M=q}^{P} \frac{1}{(M-q+1) !} \frac{\sigma(P-M+q-1, q-1)}{(P-M+q-1) !} \bar{g}_{N-P-1}=\sum_{P=q}^{N} \frac{\sigma(P, q)}{P !} \overline{\tilde{g}}_{N-P-1}$. Moreover,

it is recalled a combinatorics identity: $\sum_{q=1}^{N} \alpha^{q}(q-1) ! S(N, q)=(-1)^{N} L_{\mathrm{i}}^{1-N}(\xi)$, where $\xi=1+1 / \alpha$, and $L_{\mathrm{i}}^{k}(x)$ is the poly-logarithm function of order $k$ and argument $x$ +. A useful poly-logarithm differential property is that: $\xi d L_{\mathrm{i}}^{k}(\xi)=L_{\mathrm{i}}^{k-1}(\xi) d \xi$. These identities allows to finally rewrite formulas (23) as:

$$
B_{N}=\tilde{g}_{N}+\frac{1}{\xi}\left[f(x, N, \xi)+\sum_{M=1}^{N-1} \overline{\tilde{g}_{N-M-1}} f(x, M, \xi)\right]
$$

I http://en.wikipedia.org/wiki/Stirling_number

+ http://en.wikipedia.org/wiki/Polylogarithm 


$$
A_{N}=\frac{\left|L_{\mathrm{i}}^{-N}(\xi)\right|}{(1+\alpha) N !}+\overline{\tilde{g}_{N-1}}+\sum_{M=1}^{N-1} \frac{\left|L_{\mathrm{i}}^{-M}(\xi)\right|}{\tilde{g}_{N-M-1}} \frac{(1+\alpha) M !}{(1+2)}
$$

with $f(x, k, \xi)=\sum_{i=1}^{k} \frac{x^{i}}{i !} \frac{\left|L_{\mathrm{i}}^{i-k}(\xi)\right|}{(k-i) !}$. Formulas (24) are now in a form that makes easier to establish uniform convergence of series $y_{N}$ to solution $y$. Infact, for $N$ large, one can neglect the terms $\tilde{g}_{N}$ and $\overline{\tilde{g}_{N-1}}$ as compared to the others. Second, the function $f(x, k, \xi)$ can be substituted with the analytical solution when $k$ is large. As such, $B_{N} \rightarrow y\left(\frac{\left|L_{\mathrm{i}}^{-N}(\xi)\right|}{(1+\alpha) N !}+\sum_{M=1}^{N-1} \frac{\left|L_{\mathrm{i}}^{-M}(\xi)\right|}{\tilde{g}_{N-M-1}}\right)$ and $B_{N} / A_{N} \rightarrow y$.

Proof of complete statement

Going back to equation (12), one sees that some assumption done previously are not satisfied: first $v=v(y)$ is a (assumed monotonic) function of $y$, second $\alpha=\alpha(x)$ and third $C=C(v, x)$ :

$$
y^{i+1}=\frac{\int_{0}^{x}\left[v\left(y^{i}\right)+\alpha(x)\right] \frac{d x}{C\left(y^{i}, x\right)}}{\int_{0}^{1}\left[v\left(y^{i}\right)+\alpha(x)\right] \frac{d x}{C\left(y^{i}, x\right)}}
$$

First of all, as done in the previous simple example, it is proven that the (limit) solution of equation (25) is unique. Infact, taking the $x$ derivative gives: $y^{\prime}=\frac{v(y)+\alpha(x)}{A C(y, x)}$, with $A=\int_{0}^{1}[v(y)+\alpha(x)] \frac{d x}{C(y, x)}$, and defining $f(y, x)=\frac{v(y)+\alpha(x)}{A C(y, x)}$, one has the ODE problem: $y^{\prime}=f(y, x), y(0)=0$. A theorem of differential equations states that if $f$ and $\partial f / \partial y$ are both continuous in $x=[0,1]$, then the solution to the ODE problem (if it exists) is unique. In this case, assuming a priori continuity of $y$, then $v(y)$ is continuous. Also $\alpha(x)$ is continuous, and as well $C(y, x)$. Moreover $\partial f / \partial y=1 /(A C)(\partial v / \partial y-A f \partial C / \partial y)$. As both $\partial v / \partial y$ and $\partial C / \partial y$ are continuous functions of $x$, it follows that the solution to the ODE is unique.

By collecting the two results: 1) that equation (25) satisfies the Arzelá-Ascoli as shown in section 3.1, as such contains at least a convergent sequence, and 2) that the fixed-point of sequence equation (25) is unique, one concludes that the convergent sequence must converge to the unique fixed-point solution $y$. 

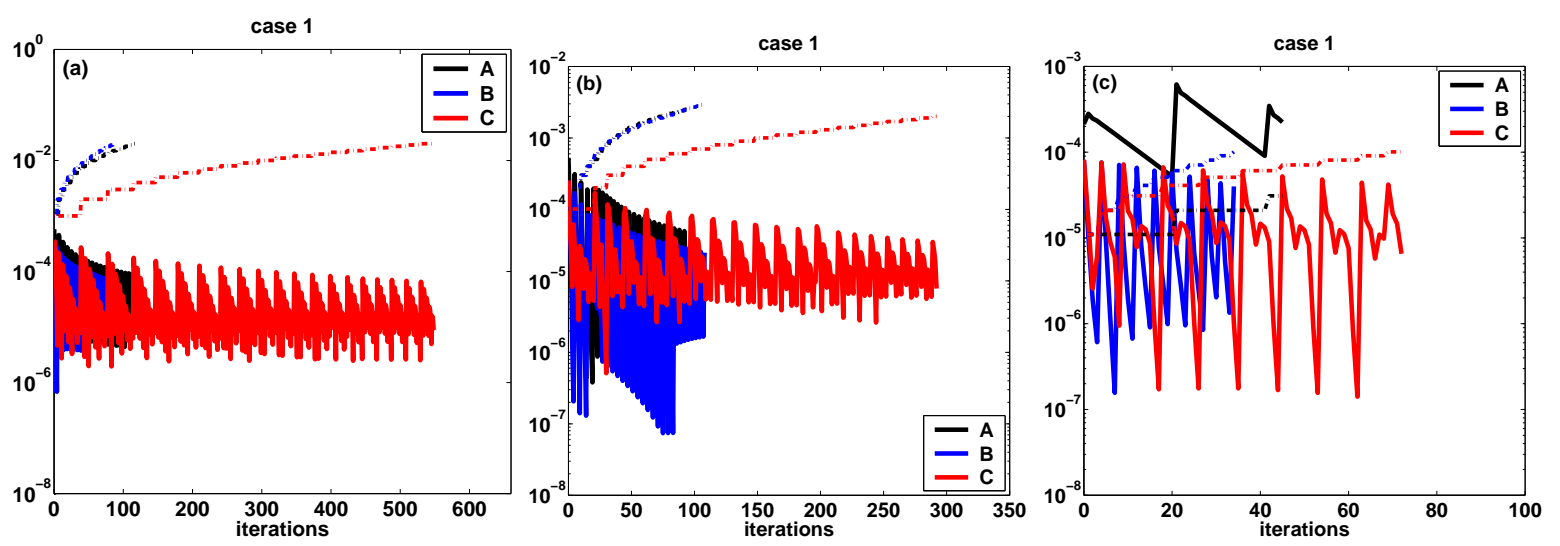

Figure 1. Case 1: relative error (solid lines) on metric coefficient $g_{2}$ during iterations, for three different choices of the coupling method (described in the text). Method A (black), Method B (blue), and Method C (red). Tolerance is set at $10^{-5}$. For each method, the dashed line is the time during the simulation in s. Plot (a) has a time step $d t=1 \mathrm{~ms}$, plot (b) has $d t=0.1 \mathrm{~ms}$, and plot (c) has $d t=0.01 \mathrm{~ms}$. 


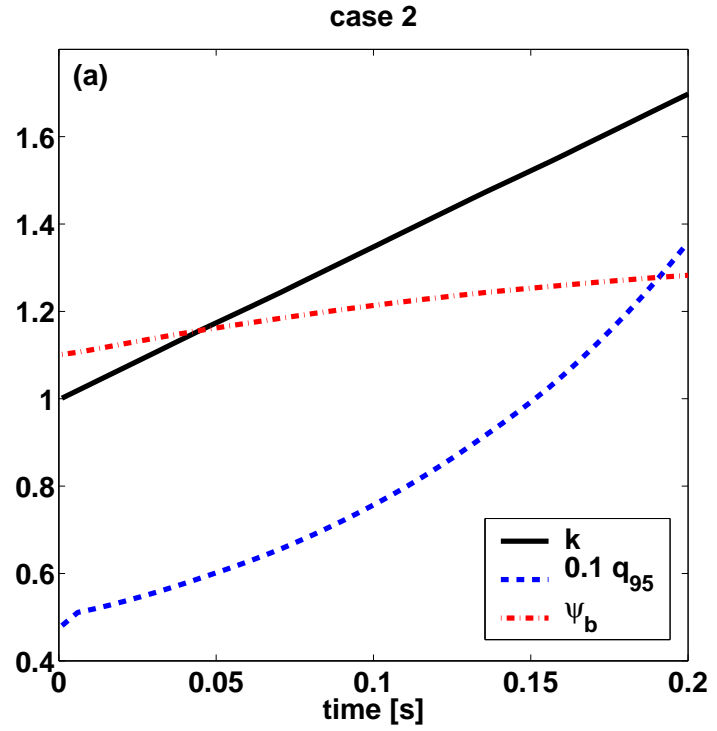

Figure 2. Case 2: time evolution of plasma elongation $k$ (solid), $q_{95}$ (rescaled by 10 , in dashed), edge poloidal magnetic flux $\psi_{\mathrm{b}}$ (dot-dashed). 

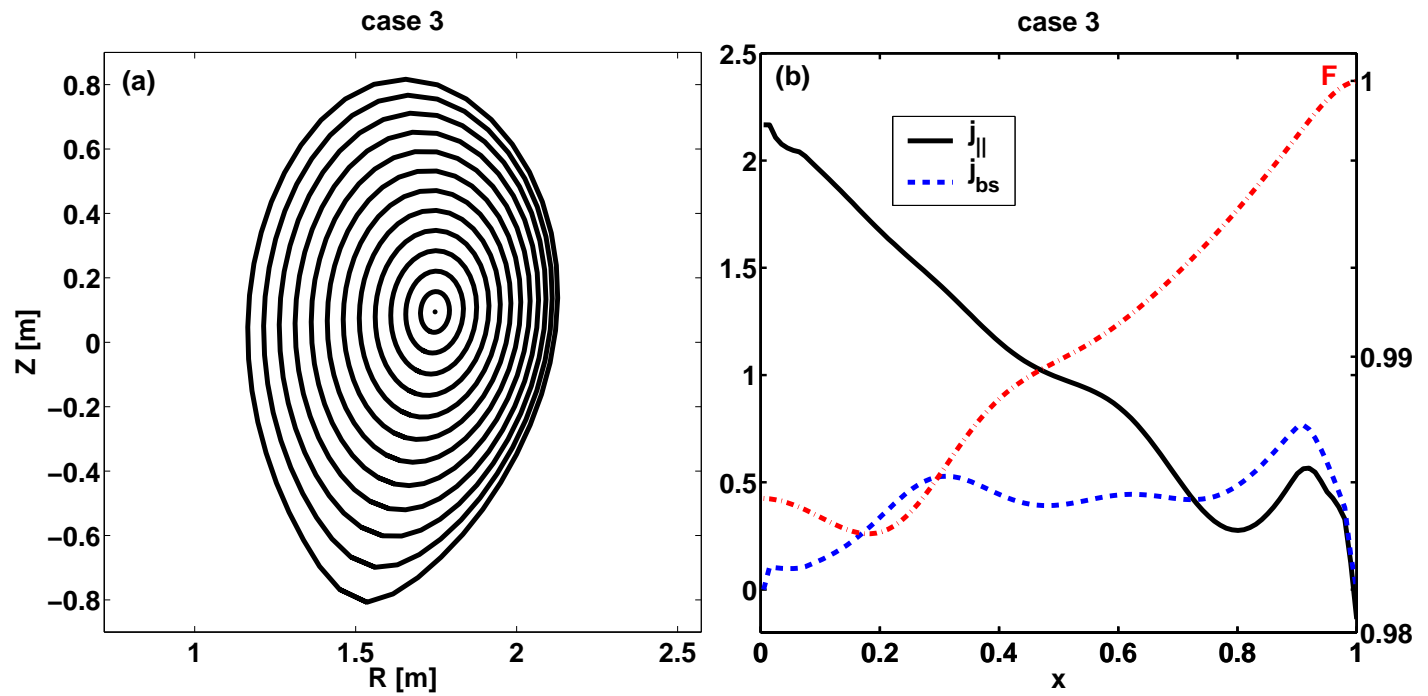

Figure 3. Case 3: a) flux surfaces shape at the last time slice. The magnetic axis Shafranov-shift is $+8 \mathrm{~cm}$ with respect to the geometrical major radius of the last closed flux surface; b) on the left $\mathrm{y}$-axis are plotted the stationary profiles of parallel current density $j_{\|}$(solid) and of the bootstrap current $j_{\text {bs }}$ (dashed) in $\left[\mathrm{MA} / \mathrm{m}^{2}\right]$. On the right $\mathrm{y}$-axis the profile of the function $F=R B_{\mathrm{T}}$ is also shown in $[\mathrm{T} \mathrm{m}]$. 

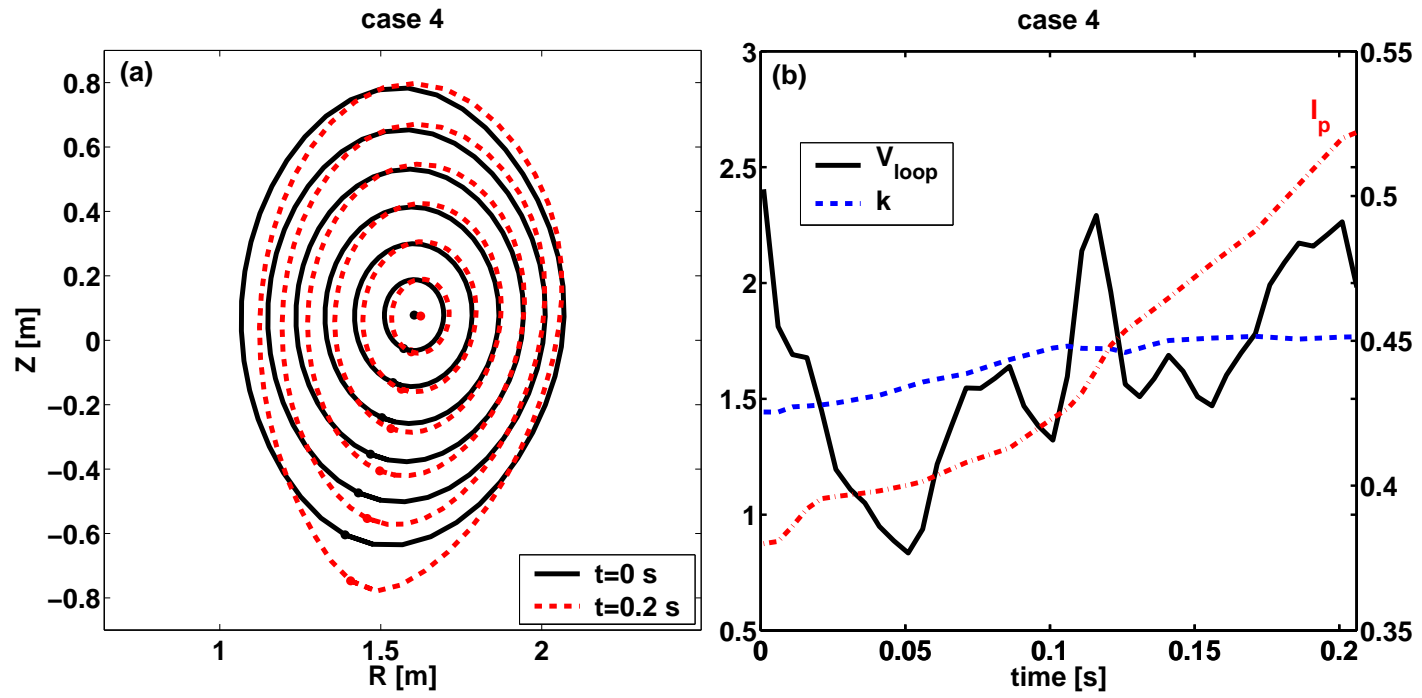

Figure 4. Case 4: a) flux surfaces shape at $t=0 \mathrm{~s}$ (solid lines) and at $t=0.2$ $\mathrm{s}$ (dashed lines); b) time evolution of plasma current $I_{\mathrm{p}}$ (dot-dashed) in [MA], boundary loop voltage $V_{\text {loop }}[\mathrm{V}]$ (solid), and boundary elongation $k$ (dashed). The lower $\mathrm{X}$-point configuration is reached at $t \approx 0.1 \mathrm{~s}$. 

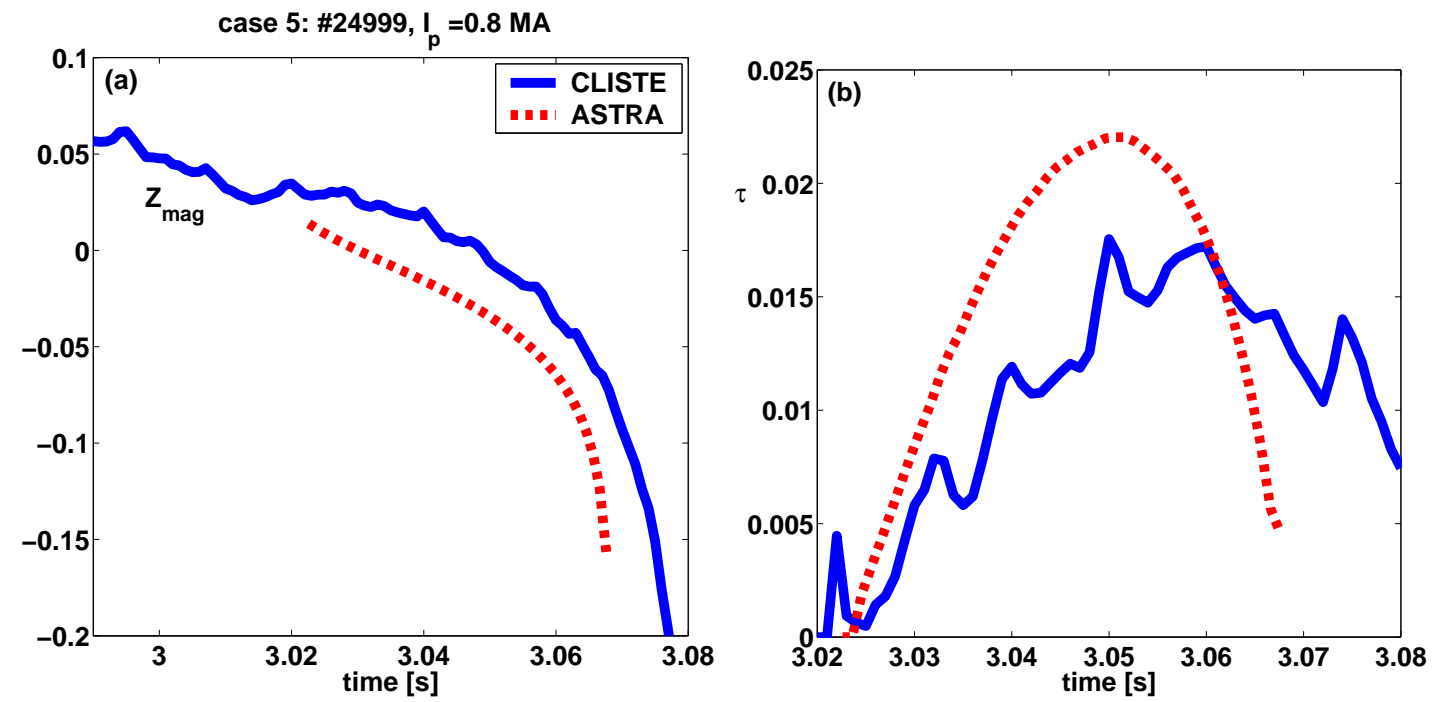

Figure 5. Case 5: a) time traces of the magnetic axis vertical position $Z_{\text {mag }}$ [m] from CLISTE (solid) and from ASTRA-SPIDER (dashed); b) comparison of istantaneous time scales $\tau=|\delta Z d t / d(\delta Z)|$, with $\delta Z=Z_{\mathrm{mag}}-Z_{\mathrm{mag}}^{t=3.02}$. 